\title{
Phenotypic characterization of human prostatic stromal cells in primary cultures derived from human tissue samples
}

\author{
GIOVANNI LUCA GRAVINA ${ }^{1,2}$, ANDREA MANCINI $^{2}$, GUIDO RANIERI $^{3}$, BORIS DI PASQUALE ${ }^{3}$, \\ FRANCESCO MARAMPON $^{1,2}$, LUIGI DI CLEMENTE ${ }^{3}$, ENRICO RICEVUTO $^{4}$ and CLAUDIO FESTUCCIA ${ }^{1}$ \\ ${ }^{1}$ Department of Biotechnological and Applied Clinical Sciences, Laboratory of Radiobiology, University of L'Aquila; \\ ${ }^{2}$ Department of Biotechnological and Applied Clinical Sciences, Division of Radiation Oncology, \\ University of L'Aquila; ${ }^{3}$ Division of Urology, Ospedale San Salvatore L'Aquila; ${ }^{4}$ Department of Biotechnological \\ and Applied Clinical Sciences, Division of Medical Oncology, University of L'Aquila, L'Aquila, Italy
}

Received September 27, 2012; Accepted November 14, 2012

DOI: 10.3892/ijo.2013.1892

\begin{abstract}
Emerging evidence has shown that the tumor microenvironment plays a crucial role in prostate cancer $(\mathrm{PCa})$ development and progression. However, the mechanism(s) through which stromal cells regulate epithelial cells and the differences among prostatic stromal cells of different histological/pathological origin in PCa progression remain unclear. Therefore, it is necessary to characterize the stromal cell populations present in benign prostatic hyperplasia (BPH) and PCa. To this end, we used cultures from stromal cells obtained from BPH-derived (15 cases) and PCa-derived (30 cases) primary cultures. In culture, stromal cells are a mixture of fibroblasts, myofibroblasts (MFs) and muscle cells. Fibroblasts are characterized for the expression of vimentin, MFs for the co-expression of $\alpha$-smooth muscle actin ( $\alpha$-SMA) and vimentin, whereas muscle cells for the expression of $\alpha$-SMA and desmin. Fibroblasts were present in large amounts in the $\mathrm{BPH}$ - compared to the $\mathrm{PCa}$-derived cultures, whereas MFs were more representative of $\mathrm{PCa}$ - as opposed to $\mathrm{BPH}$-derived cultures. Some $\alpha$-SMA-positive cells retained the expression of basal cytokeratin K14. This population was defined as myoepithelial cells and was associated with senescent cultures. The percentage of MFs was higher in high-grade compared to moderate- and low-grade PCa-derived cultures, whereas the number of myoepithelial cells was lower in high-grade compared to moderate- and low-grade PCa-derived cultures. In addition, we analyzed the expression of p75NTR, as well as the expression of matrix metalloproteinase (MMP)-2, MMP-9 and tissue inhibitors of MMPs (TIMPs). p75NTR expression was elevated in the stromal cultures derived from $\mathrm{PCa}$ compared to those derived from BPH and in cultures derived
\end{abstract}

Correspondence to: Dr Claudio Festuccia, Department of Biotechnological and Applied Clinical Sciences, Laboratory of Radiobiology, University of L'Aquila, Via Vetoio, Coppito 2, 67100 L'Aquila, Italy E-mail: festucci@univaq.it

Key words: cancer-associated fibroblasts, prostatic stromal cells, p75NTR, tumor progression from cases with Gleason scores $\geq 7$ compared to those derived from cases with Gleason scores $<7$, as well as in cultures with a high concentration of MFs compared to those with a high concentration of fibroblasts. MMP-2 was secreted by all primary cultures, whereas MMP-9 secretion was observed only in some PCa-derived stromal cells, when the percentage of MFs was significantly higher compared to BPH-derived cultures. TIMP1, TIMP2 and TIMP3 were secreted in elevated amounts in the BPH- compared to the PCa-derived stromal cultures, suggesting the differential regulation of extracellular matrix (ECM) degradation. When we used 22rv1 and PC3 $\mathrm{PCa}$ xenograft models for the isolation and characterization of murine cancer-associated fibroblasts (CAFs) we noted that the angiogenic wave was concurrent with the appearance of a reactive stroma phenotype, as determined by staining for $\alpha$-SMA, vimentin, tenascin, calponin, desmin and Masson's trichrome. In conclusion, MF stromal cells from PCa participate in the progression and metastasis of $\mathrm{PCa}$, modualting inflammation, angiogenesis and epithelial cancer cell proliferation.

\section{Introduction}

The study of prostate carcinogenesis and tumor progression is difficult due to the lack of appropriate in vitro and in vivo models. Emerging evidence has shown that the tumor microenvironment plays a crucial role in prostate cancer (PCa) development/ progression (1-4). Specifically, it has been observed that in $\mathrm{PCa}$ three disparate cellular outcomes predominate: i) the tumor remains well differentiated and clinically indolent; in this case the local stromal cells may act to restrain the growth of the cancer; ii) early in its genesis the tumor acquires a highly malignant phenotype, growing rapidly and displacing the original stromal population (often referred to as small-cell PCa); these less common aggressive tumors are relatively independent of the local microenvironment; and iii) the tumor co-opts the local stroma, taking on a classic stromagenic phenotype where interactions with the local microenvironment are critical to cancer growth. Therefore, tumor stromal cells can support tumor epithelial cell growth through the secretion of paracrine growth factors and/or providing a more favorable microenvironment 
for tumor growth. The stroma can elicit instructive, permissive, or inductive (reactive) effects on the parenchymal epithelium. For example, embryonic mesenchymal cells can 'instruct' epithelial cells to form functional, differentiated glands. By contrast, a 'permissive' stroma supports a previously-induced epithelial phenotype (5-8).

Molecular markers of stromal cell subpopulations have been poorly defined. Generally, fibroblasts are identified by their spindle-shaped morphology and the overlapping expression of various 'indicators' such as vimentin. Stroma cells are androgen receptor (AR)-negative. However, some cells retain the expression of AR $(9,10)$. Stromal AR regulates epithelial proliferation, extracellular matrix (ECM) remodelling, neovasculature formation and immune cell infiltration through the secretion of pro-inflammatory cytokines/chemokines, whereas the loss of stromal AR leads to suppressed prostate tumorigenesis $(9,11)$. Transforming growth factor- $\beta$ (TGF- $\beta$ ) is a growth factor abundantly present in the stroma. This can induce or suppress differentiation and tumorigenesis in a dose- and context-dependant manner (4). Loss of the TGF- $\beta$ type II receptor (TGF $\beta R 2$ ) has been observed in the stroma of $>60 \%$ of human PCa patients (12). Neurotrophin growth factor [nerve growth factor (NGF), brain-derived neurotrophic factor (BDNF), neurotrophin-3 (NT-3) and neurotrophin-4/5 (NT-4/5)] binding activates tyrosine kinase receptors encoded by the Trk family (Trk A, Trk B and Trk C), and the low-affinity receptor p75NTR. In the normal prostate, p75NTR and Trk A are found on benign prostate epithelial cells, whereas NGF- $\beta$ is expressed by adjacent stromal cells (13-16). NGF- $\beta$ stimulates the growth of prostate epithelial cells, which suggests the paracrine regulation of prostate cell growth by NGF- $\beta$ or other neurotrophins. In contrast to benign prostate epithelial cells, p75NTR expression is decreased in cancer cells $(13,14)$. A dramatic increase in p75 immunoreactivity has been observed in the stroma and this correlates with an increase in malignancy (16).

To better clarify the cellular interactions mediated by the reactive stroma, we characterized the stromal cell population present in benign prostatic hyperplasia (BPH) and PCa using a previously described method $(17,18)$. To this end, we evaluated the expression of vimentin (in fibroblasts), vimentin and $\alpha$-smooth muscle actin [ $\alpha$-SMA; in myofibroblasts (MFs)] or desmin (in muscle), as well as the expression of p75NTR, matrix metalloproteinase (MMP)-2 and MMP-9 and their tissue inhibitors (TIMPs). Stromal cells are a mixture of fibroblasts and MFs $(19,20)$. The number of fibroblasts was higher in $\mathrm{BPH}$ - compared to PCa-derived cultures, whereas the number of MFs was higher in PCa- compared to BPH-derived cultures. The percentage MFs was higher in high-grade PCa-derived cultures compared to moderate- and low-grade PCa-derived cultures. p75NTR expression was also elevated in stromal cell cultures derived from PCa compared to those derived from $\mathrm{BPH}$ and in cultures derived from cases with Gleason scores $\geq 7$ compared to those derived from cases with Gleason scores $<7$, as well as in cultures with a high concentration of MFs (vimentin/ $\alpha$-SMA/desmin-positive) compared to cultures with a high concentration of fibroblasts. The present study confirms a novel, malignant-dependent localization of p75 in smooth muscle cells SMCs/MFs in stromal cell cultures derived from human PCa. Therefore, p75 re-expression in stromal SMCs/
MFs is a mechanism related to the general dedifferentiation of the stroma connected to neoplastic invasion.

\section{Materials and methods}

Reagents. All materials for tissue culture were purchased from HyClone (Cramlington, NE, USA). Plasticware was obtained from Nunc (Roskilde, Denmark). Antibodies, when not defined otherwise, were purchased from Santa Cruz Biotechnology, Inc. (Santa Cruz, CA, USA). Elisa kits for TGF- $\beta 1$ and stromal cell-derived factor $\alpha$ (SDF1 $\alpha)$ were purchased from RayBiotech (European distributor, Hölzel Diagnostika GmbH, Köln, Germany).

Stromal cell cultures from prostatic specimens. Tissue samples were collected by radical prostatectomy from patients attending the Urology Division of San Salvatore Hopital, L'Aquila, Italy. Our Institutional review board approved the protocol and a written informed consent was obtained from all human subjects. The tissue samples were enzymatically digested with collagenase type I (225 U/ml; Sigma, St. Louis, MA, USA) and hyaluronidase (125 U/ml; Sigma) in DMEM (with 10\% FCS) at $37^{\circ} \mathrm{C}$ overnight. The supernatant containing the stromal cells was centrifuged at $250 \mathrm{x} \mathrm{g}$ for $5 \mathrm{~min}$. The pellet was resuspended and plated in complete DMEM. After allowing the cells to grow to confluence, they were briefly trypsinized to release the stromal cells, which were then removed and expanded in DMEM with 5\% FCS. After five passages, a homogeneous stromal cell population was established. Microscopy and immunocytochemistry were used to determine the purity of the stromal cell fraction. Epithelial cell exclusion assay was performed using a panel of different antibodies purchased from Sigma, when not specified. In particular, we used an anti-PAN cytokeratin antibody to evaluate the epithelial presence, anti$\mathrm{K} 18$, clone CY90, anti-K8 and clone M20 to evaluate luminar cells and anti-K14, clone CKB1 and anti-K5 (N20) polyclonal antibody to recognize basal cells. Anti-desmin polyclonal antibody was used to determine smooth muscle cell presence. Anti $\alpha$-SMA, clone 1A4, anti-vimentin and clone V9, were used to determine the desmin-negative MF and fibroblast cell populations. Cells were counted and expressed as a percentage of vimentin-positive cells per microscopic field at x200 magnification. Five separate fields were considered and the percentage of positive cells was expressed as the mean $\pm \mathrm{SE}$.

Western blot analysis. Cells were grown to confluence in $100-\mathrm{mm}$ dishes. The medium was then removed and the cells were washed with cold PBS and immediately lysed with $1 \mathrm{ml}$ of lysis buffer (50 mM HEPES, pH 7.5, $150 \mathrm{mM} \mathrm{NaCl}$, $10 \%$ glycerol, $1 \%$ Triton X-100, 1 mM EDTA, 1 mM EGTA, $50 \mathrm{mM}$ NaF, $30 \mathrm{mM}$ p-nitrophenyl phosphate, $10 \mathrm{mM}$ sodium pyrophosphate, $1 \mathrm{mM}$ phenylmethylsulfonyl fluoride, $10 \mu \mathrm{g} / \mathrm{ml}$ aprotinin and $10 \mu \mathrm{g} / \mathrm{ml}$ leupeptin). Lysates were centrifuged at $10,000 \times \mathrm{g}$ for $10 \mathrm{~min}$. Equal amounts of protein $(50 \mu \mathrm{g})$ were resolved using 7.5\% SDS-polyacrylamide gel electrophoresis (SDS-PAGE). After electrophoresis, separated proteins were blotted onto a nitrocellulose membrane and analyzed by western blot analysis with the ECL method. Proteins were transferred from the gel onto a nitrocellulose filter paper. The membrane was washed with saline solution (Tris- $\mathrm{HCl}, \mathrm{NaCl}$ and BSA at 

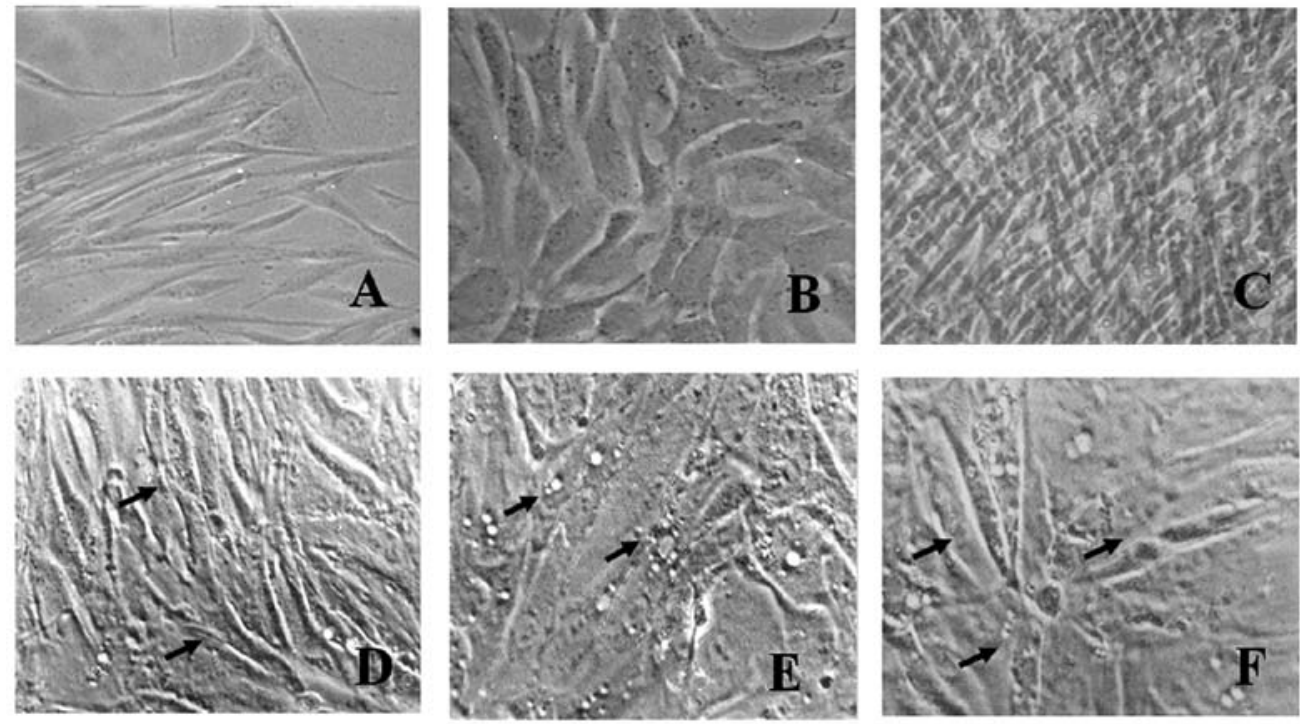

Figure 1. Phase contrast photomicrograph of stromal cell cutlures. (A-F) x200 images. (A-C) Visible fibroblastic-like cells at different densities of growth ; (D-F) elongated cells with a final tendency to form fused cells (arrows, D and F) with several bright cytoplasmic organelles (arrows, E). The cells resemble myoepithelial/myofibroblast cells.

$1 \%)$ for $1 \mathrm{~h}$ and then incubated with different antibodies diluted at $1 \mu \mathrm{g} / \mathrm{ml}$. After incubation the membrane was washed with Tris-NaCl plus $0.1 \%$ Tween-20 and incubated for $1 \mathrm{~h}$ with a second antibody conjugated with peroxidase $(1: 5,000)$. Protein bands were quantified by densitometry. Immunoreactive bands were visualized using enhanced chemiluminescence detection kit reagents (Amersham, Milan, Italy).

Gelatin zymography. The zymography assay (21) used gelatin as a substrate for MMP-2 and MMP-9. Gelatin at a concentration of $0.1 \%$ was incorporated into a $10 \%$ polyacrylamide gel containing $0.4 \%$ SDS. Electrophoresis under non-reducing conditions was performed using the Bio-Rad mini-gel system at $125 \mathrm{~V}$ for 90-120 min. For plasminogen activator analysis, SDS-polyacrilamide gels were polymerized with $0.1 \mathrm{mg} / \mathrm{ml}$ of lactose-free casein and $15 \mu \mathrm{g} / \mathrm{ml}$ of human plasminogen as previously described (22). After electrophoresis the gels were washed twice for $30 \mathrm{~min}$ in $2.5 \%$ Triton X-100 (v/v) to remove the SDS and then incubated overnight in the developing buffer [50 mM Tris- $\mathrm{HCl}$ (pH 7.6), $200 \mathrm{mM} \mathrm{NaCl}, 5 \mathrm{mM} \mathrm{CaCl}_{2}, 0.2 \%$ (v/v) Brij-35] at $37^{\circ} \mathrm{C}$. Digestion bands were quantified by the Java image processing program 'ImageJ' from the scanned gels. Reverse zymography for TIMPs was performed in concentrated supernatants containing $20 \mu \mathrm{g}$ of proteins [conditioned medium concentrated 20 -fold by Centricon (Amicon, Beverly, MA, USA) with $10 \mathrm{kDa}$ molecular weight cut-off] analyzed by $14 \%$ SDS-PAGE containing 0.1 casein and $30 \%$ $(\mathrm{v} / \mathrm{v})$ serum-free supernatants from the MDA-MB-231 human breast cancer cell line. This cell line expresses a single $53 \mathrm{kDa}$ band of EDTA-inhibitable caseinolytic activity in zymograms (MMP-3). Gels were processed as described above except for a 72-h incubation in collagenase buffer. Dark stained bands of 28-25 and $21 \mathrm{kDa}$ molecular weight were identified as inhibitor bands of TIMP1/TIMP3 and TIMP2, respectively.

Statistics. The data are expressed as the mean and standard deviation (SD) and were compared using an unpaired Student's t-test. Categorical data were analyzed by the exact Fisher's test. A p-value $<0.05$ was considered to indicate a statistically significant difference.

\section{Results}

We characterized a total of 45 primary cultures derived from clinical samples, which, after the completion of the clinical work-up for each patient, were grouped according to the pathological diagnosis formulated through the microscopic analysis of the biopsies obtained in parallel with cultured tissue samples. We studied in primary culture 15 cases of BPH and 30 cases of $\mathrm{PCa}$ of various pathological grades.

Stromal cell characterization. Primary cell culture was characterized at each passage and a gradual decrease in the number of rounded cells with epithelial appearance and an increase in the number of elongated cells with fibroblastic aspects was observed, as demonstrated by the phase contrast micrographs (Fig. 1). In previous studies, it has been demonstrated that prostatic stromal cells are characterized by an increase in the expression of cytoskeleton proteins, such as vimentin and $\alpha$-SMA, followed by a decrease in muscle markers, such as desmin $(23,24)$. After five passages in our culture system, a cell population positively expressing vimentin and $\alpha$-SMA, but negatively expressing cytokeratin 18 and weakly expressing desmin was obtained. These results indicate that our human pluripotent stem cell populations represent a combination of fibroblasts (vimentin-positive cells) and MFs ( $\alpha$-SMA-positive cells). Fig. 2 demonstrates the expression of cytoskeleton markers ( $\alpha$-SMA, vimentin and desmin) by western blot analysis. Fig. 3 demonstrates the expression of stromal markers, thymidine phosphorylase (TP), as well as that of the prostate markers, AR and prostate-specific antigen (PSA), or caveolin-1 and tenascin $\mathrm{C}$ as shown by western blot analysis in different cultures derived from BPH and PCa samples. The amount of these markers was quantified densitometrically as arbitrary densitometric units (ADUs) after normalization with 
A $\mathrm{BPH}$-derived cultures

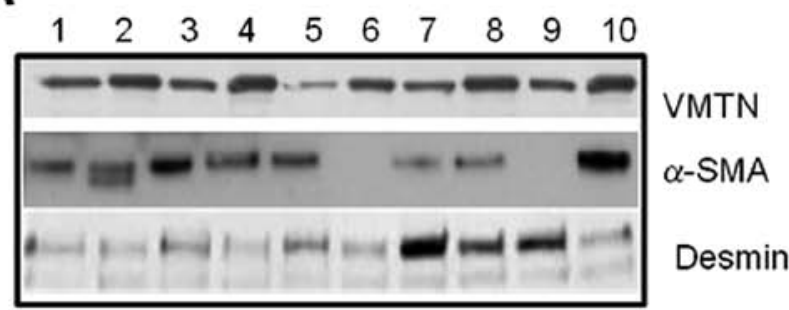

\begin{tabular}{|c|c|c|c|}
\hline case & VMTN $(\%)$ & $\begin{array}{l}\alpha \text {-SMA } \\
(\%)\end{array}$ & $\begin{array}{l}\text { Desmin } \\
(\%)\end{array}$ \\
\hline 1 & $43+1-2$ & $20+1.2$ & $10+1-2$ \\
\hline 2 & $60+1-8$ & $35+1.5$ & $10+1-2$ \\
\hline 3 & $40+1-5$ & $40+1.4$ & $15+1-2$ \\
\hline 4 & $65+1-8$ & $44+1.5$ & $10+1-3$ \\
\hline 5 & $27+1-5$ & $42+1.5$ & $20+1-2$ \\
\hline 6 & $60+1-4$ & $<5$ & $10+1-2$ \\
\hline 7 & $44+1-3$ & $15+1-2$ & $45+1.5$ \\
\hline 8 & $70+1-11$ & $25+1.4$ & $30+1-4$ \\
\hline 9 & $55+1.5$ & $<5$ & $35+1-5$ \\
\hline 10 & $85+1-6$ & $30+1.4$ & $15+1-2$ \\
\hline
\end{tabular}

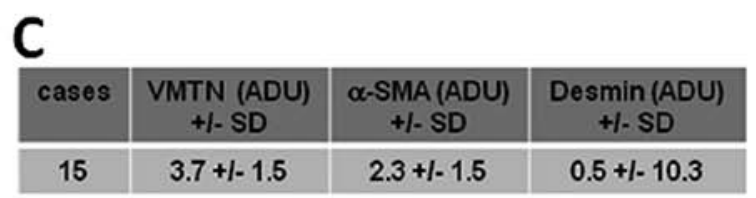

D

\begin{tabular}{|c|c|c|c|}
\hline cases & $\begin{array}{c}\text { VMTN (\%) } \\
+l-S D\end{array}$ & $\begin{array}{c}\alpha \text {-SMA (\%) } \\
+l-S D\end{array}$ & $\begin{array}{c}\text { Desmin }(\%) \\
+l-S D\end{array}$ \\
\hline 15 & $\mathbf{5 4 . 3 + l - 1 6 . 0}$ & $\mathbf{2 4 . 1 + l - 1 4 . 4}$ & $\mathbf{1 7 . 3 + l - 1 2 . 3}$ \\
\hline
\end{tabular}

PCa-derived cultures

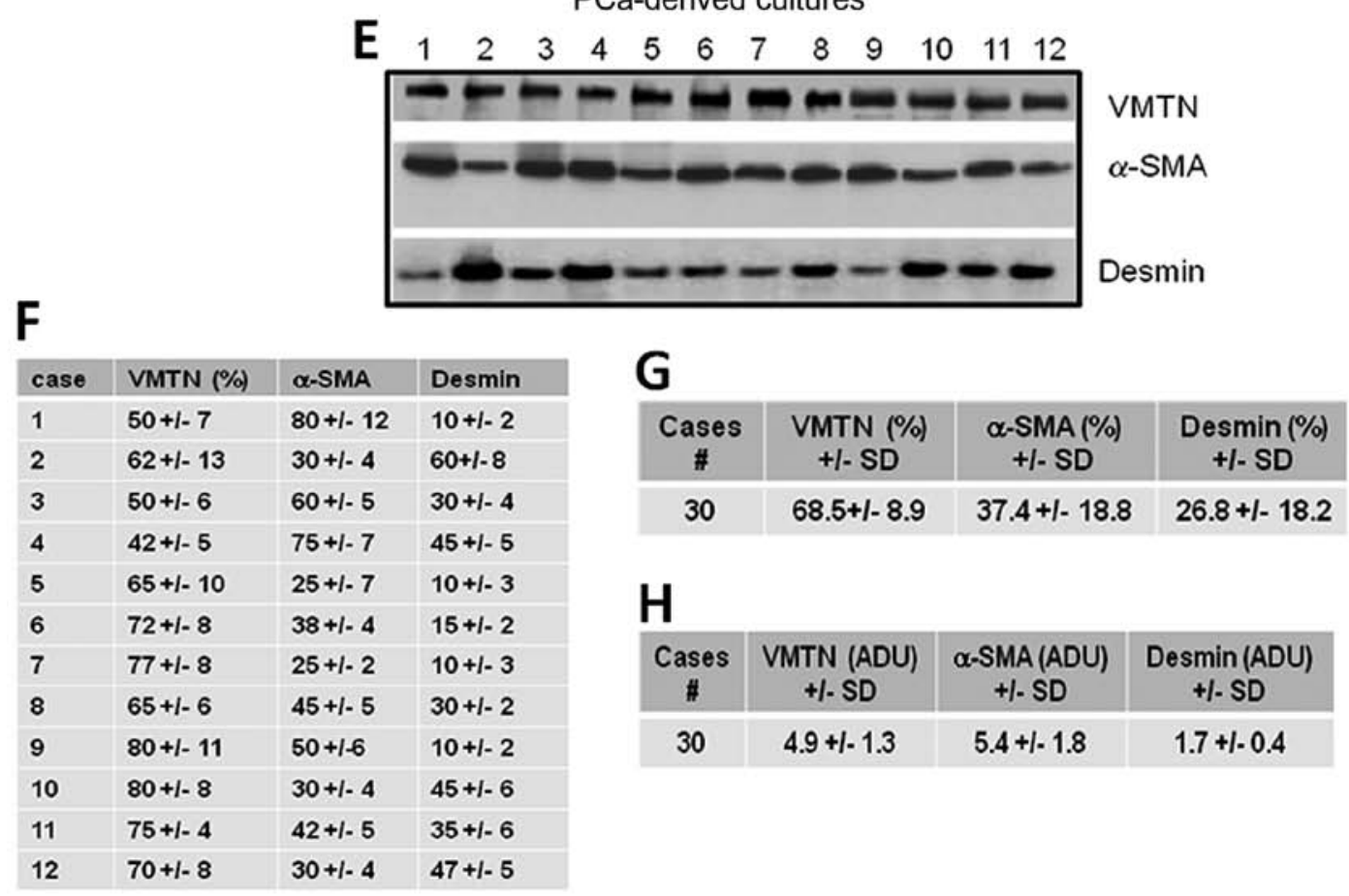

Figure 2. Western blot analyses of stromal cell markers, vimentin, desmin and $\alpha$-muscle actin ( $\alpha$-SMA), in primary culture cell extracts. (A) Representative BPH-derived 10 cultures. (B) Percentage of vimentin (VMTN)-, desmin- and $\alpha$-SMA-positive cells in BPH-derived 10 cultures analyzed by western blot analysis. (C) Mean percentage of VMTN, $\alpha$-SMA and desmin in the total 15 cases of BPH. (D) Adjusted densitometric units obtained after normalization to actin levels of immunoreactive bands in western blot analysis in the 15 BPH-derived cultures. (E) Representative PCa-derived 12 cultures. (F) Percentage of VMTN-, desmin- and $\alpha$-SMA-positive cells in BPH-derived 12 cultures analyzed by western blot analysis. (G) Adjusted densitometric units obtained after normalization to actin levels of immunoreactive bands in western blot analysis in the 30 PCa-derived cultures. (H) Mean percentage of VMTN, $\alpha$-SMA and desmin in the 30 cases of PCa. Each lane was loaded with $40 \mu \mathrm{g}$ of total protein.

$\beta$-actin. Vimentin expression was not statistically different in the BPH- compared to the PCa-derived cultures, both as the percentage of positve cells and adjusted densitometric units. Although the percentage of $\alpha$-SMA-positive cells (Fig. 2B, C and $\mathrm{G}$ ) was not statistically significant, the protein levels were significantly higher in the $\mathrm{PCa}$ - compared to the $\mathrm{BPH}$-derived cultures $(\mathrm{p}<0.001$, Fig. 2A, D and H). Similar results were obtained with desmin; although the percentage of desminpositive cells (Fig. 2B, C and G) was not statistically significant, the protein levels were significantly higher in $\mathrm{PCa}$ - compared to $\mathrm{BPH}$-derived cultures. Desmin expression was weakly and mainly associated with PCa stromal cultures ( $p<0.001$, Fig. 2A, 


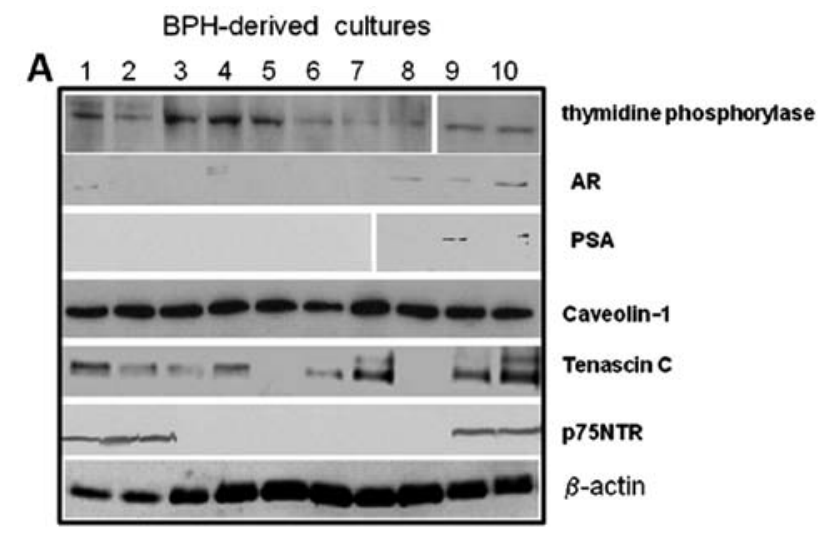

PCa-derived cultures

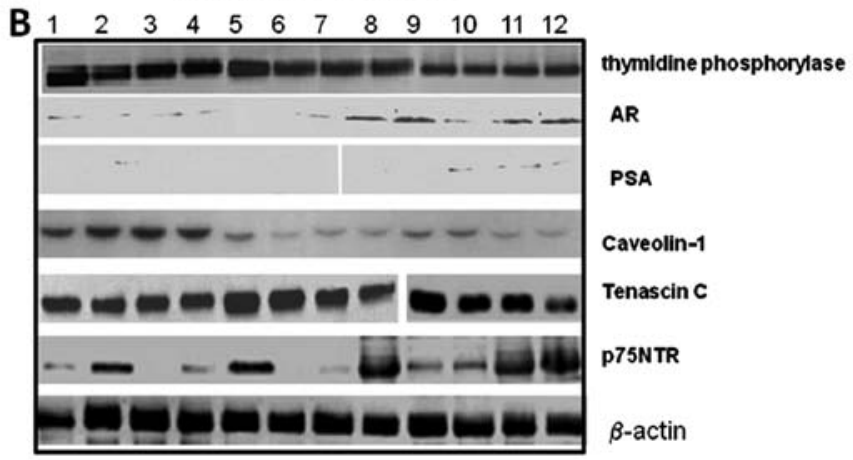

Figure 3. Western blot analyses of thymidine phosphorylase (TP), androgen receptor (AR), prostate-specific antigen (PSA), caveolin-1, tenascin $\mathrm{C}$ and p75NTR in primary culture cell extracts harvested from (A) BPH- and (B) PCa-derived cultures. Each lane was loaded with $40 \mu \mathrm{g}$ of total protein.

$\mathrm{D}$ and $\mathrm{H})$. Although the number of samples was limited, we aimed to determine the correlation between the stromal cell population and histological grade (Gleason grade) in our samples. No statistically significant differences were observed for vimentin expression $[2.1 \pm 1.0(\mathrm{G} 2-4)$ ADU vs. $2.7 \pm 1.5$ (G5-7) ADU and 4.3 \pm 1.8 (G8-10) ADU] and for $\alpha$-SMA expression [4.7 $\pm 1.2(\mathrm{G} 2-4)$ ADU vs. $4.9 \pm 1.7$ (G5-7) ADU and 5.7 \pm 2.1 (G8-10) ADU]. Desmin expression was significantly lower in cultures derived from cases with a low Gleason grade compared to those derived from cases with a high Gleason grade $[0.4 \pm 0.2$ (G2-4) ADU vs. $1.3 \pm 0.4$ (G5-7) ADU and 2.4 \pm 0.6 in (G8-10), $\mathrm{p}<0.01]$. The expression of K14 was observed in some stromal primary cultures and was restricted to $\mathrm{PCa}$ - compared to $\mathrm{BPH}$-derived cultures (immuno-reactive bands for K14 were found in 1/15 BPH and 12/30 PCa cases; not significant) and in high-grade compared to moderate- and low-grade samples [1/7 (14.3\%, G2-4), 5/15 (33.3\%, G5-7) and 6/8 (75\%, G8-10), $\mathrm{p}=0.043]$.

The levels of tenascin $\mathrm{C}$ verified by western blot analysis using the Santa Cruz Biotechnology (Scbt) antibody, N19, were significantly higher in cultures derived from $\mathrm{PCa}$ samples [1.2 $\pm 0.9 \mathrm{ADU}($ mean $\pm \mathrm{SD})$ and $4.8 \pm 0.8$ for $\mathrm{BPH}$ - and Ca-derived cultures, respectively, $\mathrm{p}<0.001]$ and in high-grade compared to moderate- and low-grade samples $[3.2 \pm 0.4$ (G2-4), $3.9 \pm 0.1$ (G5-7) and 6.0 \pm 1 (G8-10), $\mathrm{p}<0.001]$.

The AR (Scbt N20) is expressed in a subset of prostatic stromal cells and functional stromal cell AR is required for normal prostate development and influences the growth of prostate tumors. Fibroblasts are negative for AR, whereas MFs express AR. We observed that AR expression was present only in some PCa-derived cultures $(17 / 30,56.7 \%$, vs. $5 / 15$, $33.3 \%$, in PCa- and BPH-derived cultures, respectively) and in high-grade compared to moderate- and low-grade samples [3/10 (G2-4, 30\%) vs. 7/15 (G5-7, 40\%) and 7/8 (G8-10, 87.5\%), $\mathrm{p}<0.005]$. PSA secretion (Scbt A67-B/E3) was extremely low/absent in BPH- and PCa-derived cultures. The levels of p75NTR were significantly higher in PCa-derived (2.5 \pm 2.0 ADU) compared with BPH-derived cultures $(0.8 \pm 0.7 \mathrm{ADU}$, $\mathrm{p}<0.005)$ and in high-grade compared to moderate- and low-grade samples $[0.6 \pm 0.5$ (G2-4) ADU vs. 2.1 \pm 1.4 (G5-7) ADU and $4.7 \pm 0.8$ in $(G 8-10), p<0.001]$. Since changes in the levels of stromal constituents, such as reduced levels or loss of caveolin-1 expression and increased TP (Imgenex, San Diego, CA) levels, were associated with PCa-derived cultures with high Gleason scores compared to BPH with low Gleason scores, we analyzed the expression of these proteins. We observed that caveolin-1 (Scbt H96) levels were significantly higher in BPH- compared to PCa-derived cultures [3.3 \pm 0.5 ADU for BPH vs. $1.5 \pm 0.9 \mathrm{ADU}$, respectively; $\mathrm{p}<0.001]$ and in low-grade compared to moderate- and high-grade samples [2.6 \pm 0.4 (G2-4) ADU vs. $1.2 \pm 0.2$ (G5-7) ADU and 0.7 \pm 0.2 in (G8-10); $\mathrm{p}<0.005]$. TP levels were significantly lower in $\mathrm{BPH}-$ compared to PCa-derived cultures [0.8 $\pm 0.5 \mathrm{ADU}$ for $\mathrm{BPH}$ vs. $3.5 \pm 0.7 \mathrm{ADU}$, respectively; $\mathrm{p}<0.001$ ], whereas no statistically significant differences were observed for low-, moderate- and high-grade PCa-derived cultures $[3.3 \pm 0.5$ (G2-4) ADU vs. 4.1 \pm 0.3 (G5-7) ADU and 3.2 \pm 0.8 in (G8-10); not significant].

We then analyzed the secretion of two major stromal secreted cytokines (SDF1 $\alpha$ and TGF- $\beta$ ) in conditioned media harvested from subconfluent $\mathrm{BPH}-$ and $\mathrm{PCa}$-derived cultures. The results demonstrated that the levels of TGF- $\beta 1$ and SDF1 $\alpha$ were significantly higher in PCa-derived cultures $(65.0 \pm 27.8 \mathrm{pg} /$ $\mathrm{ml}$ for SDF $1 \alpha$ and $158 \pm 64 \mathrm{pg} / \mathrm{ml}$ for TGF- $\beta 1$ ) compared to those observed in BPH-derived cultures $(12 \pm 4 \mathrm{pg} / \mathrm{ml}$ for SDF1 $\alpha$ and $21 \pm 6 \mathrm{pg} / \mathrm{ml}$ for TGF- $\beta 1 ; \mathrm{p}<0.001$ for both cytokines). SDF1 $\alpha$ and TGF- $\beta 1$ secretion was higher and with respect to moderateand low-grade samples [SDF1 $\alpha$ : 40.3 $\pm 12.0(\mathrm{G} 2-4) \mathrm{ADU}$ vs. 56.5 \pm 15.3 (G5-7) ADU and 72.7 \pm 31.2 (G8-10), $\mathrm{p}<0.005$; TGF- $\beta 1$ : $84.6 \pm 37.0$ (G2-4) ADU vs. $115.3 \pm 45.3$ (G5-7) ADU and $184.8 \pm 51.2(\mathrm{G} 8-10), \mathrm{p}<0.001]$.

Gelatin and plasminogen-dependent zymography. Fig. 4 demonstrates that prostatic stromal cell cultures primarily secreted MMP-2, whereas MMP-9 secretion was low/absent in BPH-derived cultures (Fig. 4A) and restricted in some PCa-derived cultures (Fig. 4B; 12/30, 40\%). Higher levels of the active form of MMP-2 were observed in PCa- compared to BPH-derived cultures.

TIMP1, TIMP2 and TIMP3 were also present in the conditioned media. BPH- and PCa-derived cultures (Fig. 4B) presented similar levels of TIMP1 (Fig. 4B), whereas the levels of TIMP2 were higher in PCa-derived cultures and TIMP3 was only expressed in BPH-derived cultures.

Low levels of urokinase plasminogen activator (uPA) and absent tissue levels of tissue plasminogen activator (tPA) were observed in conditioned media from BPH-derived cultures (Fig. 4A), whereas higher levels of uPA (both as high molecular 


\section{$\mathrm{BPH}$-derived cultures}
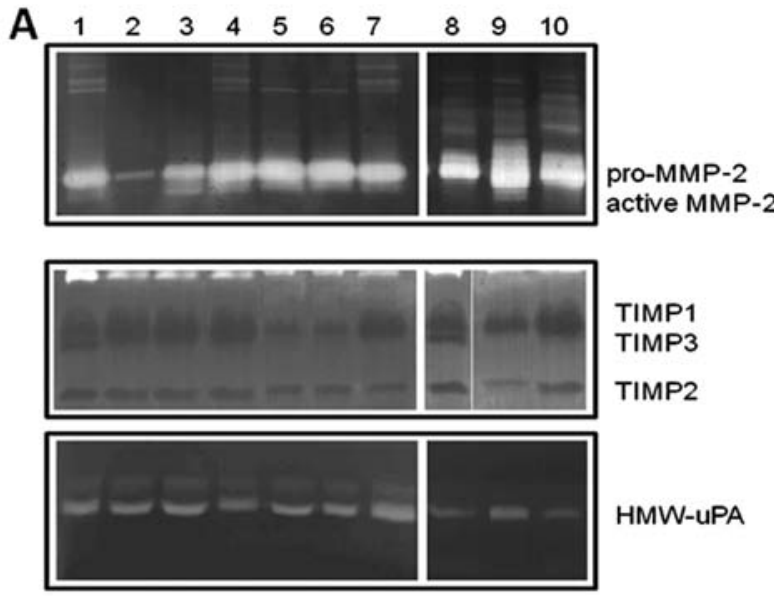

PCa-derived cultures
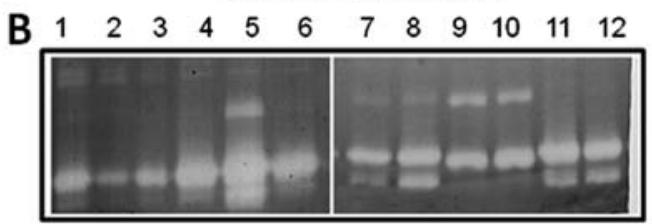

pro-MMP-9

pro-MMP-2 active MMP-2

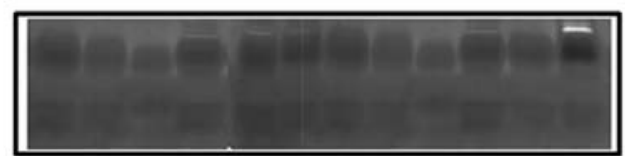

TIMP1

TIMP2

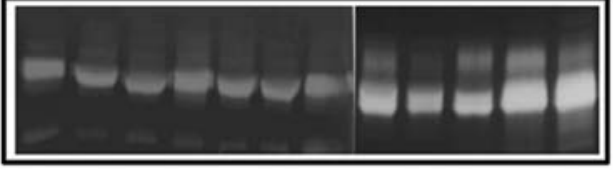

tPA

HMW-UPA

LMW-UPA

Figure 4. Gelatinolytic activity (MMP-9 and MMP-2) of tissue inhibitors of metalloproteinase (TIMP1, TIMP2 and TIMP3) and plasminogen-dependent enzymes (tPA and UPA) present in conditioned media harvested after $48 \mathrm{~h}$ of subconfluent primary stromal culture cell cultures derived from (A) BPH and (B) PCa tissue samples. Each lane was loaded with $60 \mu 1$ of conditioned medium. HMW, high molecular weight; LMW, low molecular weight.

weight and low molecular weight isoform) were observed in the conditioned media from PCa-derived cultures. tPA activity was observed in some $(8 / 30,26.7 \%)$ PCa-derived cultures. High levels of pro-MMP-9 and active MMP-2 as well as uPA and tPA were obserevd in the cultures with a higher content of MFs.

\section{Discussion}

The tumor-associated stroma is not simply a supporting element for cancer cells, but plays an important role in tumor growth, invasion and metastasis. Prostate stromal cells, and the ECM which they deposit, play a key role in restraining or promoting tumorigenesis through different mechanisms, including increased arachidonate metabolism and inflammation, promoting local tumor invasion. Eicosanoids modulate the interaction of tumor cells with various host components in cancer metastasis. Their synthesis involves the release of arachidonic acid (AA) from cellular phospholipids by phospholipase A2 (PLA2), followed by metabolism by cyclooxygenases and lipooxygenases (25). The specific nature of the human PCa stromal phenotype has been shown to be an independent clinical prognostic marker $(26,27)$, underlining the importance of paracrine interactions in human disease. The prostate tumor microenvironment is complex, including cells of many different lineages (28-31). These include, but are not limited to, smooth muscle, various types of fibroblasts, senescent stromal cells, nerves and blood vessels and a wide variety of immune and inflammatory cell types. The diversity in the types of cells that compose the tumor stroma makes it difficult to study the contribution of each component. In addition, the lack of stromal cell lines that can retain the tumor-inducing properties shown by cancer-associated fibroblasts (CAFs) in vivo represents another important issue.

The results of the present study show differences in the percentage of stromal cell populations harvested from $\mathrm{BPH}$ and PCa primary stromal cultures. The two predominant cell types in the stroma of the prostate, are SMCs and fibroblasts. Fibroblasts represent the major stromal cell type, and a higher percentage of MFs are harvested from PCa. MFs are important components of the prostatic stroma. The mutual interaction through direct cell-cell contacts or paracrine signals between cancer cells and MFs is essential for invasive growth and is translated into a poor clinical prognosis. Tumor progression is deeply influenced by epigenetic changes induced by the tumor stroma. Reduced levels or loss of caveolin-1 expression and increased TP were observed in PCa-derived cultures with high Gleason scores compared to those observed in BPH-derived cultures with low Gleason scores. Caveolin-1 was strongly associated with the presence of fibroblasts. Although it has been demonstrated that the fibroblast expression of caveolin-1 favors directional migration and invasiveness of carcinoma cells in vitro as well as in vivo, stromal caveolin-1 remodels peri- and intratumoral microenvironments to facilitate tumor invasion, correlating with increased metastatic potency $(32,33)$, current evidence indicates that the increased expression of caveolin-1 in prostate adenocarcinoma cells and the downregulation of the protein in prostate stroma, mediate progression to the castration-resistant phase of PCa (34). CAFs have been reported to promote epithelial-mesenchymal transition in cancer cells, thereby enhancing their aggressiveness and stem-like properties (7,35-38). We observed that PC3 xenograft-derived murine CAFs were enriched in caveolin-1-positive cells, suggesting that expeirmental in vivo models may be similar. The presence of a reactive stromal phenotype in PCa-derived stromal cells is associated with the retention of AR and p75NTR expression, TGF- $\beta 1$, MMP-9 and VEGF secretion, as well as the overexpression of the chemokine, SDF1 $\alpha /$ CXCL12. This phenotypic appearance can support the proliferation and invasion of PCa cell lines, such as PC3 and 22rv1. MF cultures secreted higher levels of active MMP-2 both in the pro-enzyme and active form. MMP-2 is a well-known MMP related to cancer cell invasion and metastasis; its overexpression can be induced by cytokines, growth factors and oncogenes (36-38). Some cultures of PCa-derived sromal cells express MMP-9 as a pro-enzyme (37-39). This in vitro study contributes to a better understanding of the cell composition of the reactive stromal compartment in $\mathrm{PCa}$. The isolation of CAFs from aggressive or non-aggressive PCa cell xenografts may represent tool for the study of stroma-tumor cell interactions. 


\section{References}

1. Barron DA and Rowley DR: The reactive stroma microenvironment and prostate cancer progression. Endocr Relat Cancer 19: R187-R204, 2012.

2. Ammirante M, Luo JL, Grivennikov S, Nedospasov $\mathrm{S}$ and Karin M: B-cell-derived lymphotoxin promotes castrationresistant prostate cancer. Nature 464: 302-305, 2010.

3. Iacopino F, Angelucci C and Sica G: Interactions between normal human fibroblasts and human prostate cancer cells in a co-culture system. Anticancer Res 32: 1579-1588, 2012.

4. Grubisha MJ, Cifuentes ME, Hammes SR and Defranco DB: A local paracrine and endocrine network involving TGF $\beta$, Cox-2 ROS, and estrogen receptor $\beta$ influences reactive stromal cell regulation of prostate cancer cell motility. Mol Endocrinol 26 940-954, 2012.

5. Hayward SW, Wang Y, Cao M, Hom YK, Zhang B, Grossfeld GD, Sudilovsky D and Cunha GR: Malignant transformation in a nontumorigenic human prostatic epithelial cell line. Cancer Res 61: 8135-8142, 2001.

6. Cunha GR, Hayward SW, Wang YZ and Ricke WA: Role of the stromal microenvironment in carcinogenesis of the prostate. Int J Cancer 107: 1-10, 2003.

7. Olumi AF, Grossfeld GD, Hayward SW, Carroll PR, Tlsty TD and Cunha GR: Carcinoma-associated fibroblasts direct tumor progression of initiated human prostatic epithelium. Cancer Res 59: 5002-5011, 1999.

8. Barclay WW, Woodruff RD, Hall MC and Cramer SD: A system for studying epithelial-stromal interactions reveals distinct inductive abilities of stromal cells from benign prostatic hyperplasia and prostate cancer. Endocrinology 146: 13-18, 2005

9. Ricke EA, Williams K, Lee YF, Couto S, Wang Y, Hayward SW, Cunha GR and Ricke WA: Androgen hormone action in prostatic carcinogenesis: stromal androgen receptors mediate prostate cancer progression, malignant transformation and metastasis. Carcinogenesis 33: 1391-1398, 2012.

10. Tanner MJ, Welliver RC Jr, Chen M, Shtutman M, Godoy A, Smith G, Mian BM and Buttyan R: Effects of androgen receptor and androgen on gene expression in prostate stromal fibroblasts and paracrine signaling to prostate cancer cells. PLoS One 6: e16027, 2011.

11. Lai KP, Yamashita S, Huang CK, Yeh S and Chang C: Loss of stromal androgen receptor leads to suppressed prostate tumourigenesis via modulation of pro-inflammatory cytokines/ chemokines. EMBO Mol Med 4: 791-807, 2012.

12. Li X, Sterling JA, Fan KH, Vessella RL, Shyr Y, Hayward SW, Matrisian LM and Bhowmick NA: Loss of TGF- $\beta$ responsiveness in prostate stromal cells alters chemokine levels and facilitates the development of mixed osteoblastic/osteolytic bone lesions. Mol Cancer Res 10: 494-503, 2012.

13. Delsite R and Djakiew D: Characterization of nerve growth factor precursor protein expression by human prostate stroma cells: a role in selective neurotrophin stimulation of prostate epithelial cell growth. Prostate 41: 39-48, 1999.

14. Krygier S and Djakiew D: The neurotrophin receptor p75NTR is a tumor suppressor in human prostate cancer. Anticancer Res 21: 3749-3755, 2001.

15. Festuccia C, Gravina GL, Muzi P, Pomante R, Ventura L, Ricevuto $\mathrm{E}$, Vicentini $\mathrm{C}$ and Bologna $\mathrm{M}$ : In vitro and in vivo effects of bicalutamide on the expression of TrkA and P75 neurotrophin receptors in prostate carcinoma. Prostate 67: 1255-1264, 2007.

16. Rende M, Rambotti MG, Stabile AM, Pistilli A, Montagnoli C, Chiarelli MT and Mearini E: Novel localization of low affinity NGF receptor (p75) in the stroma of prostate cancer and possible implication in neoplastic invasion: an immunohistochemical and ultracytochemical study. Prostate 70: 555-561, 2010.

17. Festuccia C, Vincentini C, di Pasquale AB, Aceto G, Zazzeroni F, Miano L and Bologna M: Plasminogen activator activities in short-term tissue cultures of benign prostatic hyperplasia and prostatic carcinoma. Oncol Res 7: 131-138, 1995.

18. Festuccia C, Angelucci A, Gravina GL, Muzi P, Miano R, Vicentini $C$ and Bologna M: Epithelial and prostatic marker expression in short-term primary cultures of human prostate tissue samples. Int J Oncol 26: 1353-1362, 2005.

19. Sugimoto H, Mundel TM, Kieran MW and Kalluri R: Identification of fibroblast heterogeneity in the tumor microenvironment. Cancer Biol Ther 5: 1640-1646, 2006.
20. De Wever O, Demetter P, Mareel M and Bracke M: Stromal myofibroblasts are drivers of invasive cancer growth. Int J Cancer 123: 2229-2238, 2008

21. Festuccia C, Angelucci A, Gravina G, Eleuterio E, Vicentini C and Bologna M: Bombesin-dependent pro-MMP-9 activation in prostatic cancer cells requires beta1 integrin engagement. Exp Cell Res 280: 1-11, 2002.

22. Festuccia C, Dolo V, Guerra F, et al: Plasminogen activator system modulates invasive capacity and proliferation in prostatic tumor cells. Clin Exp Metastasis 16: 513-528, 1998.

23. Schor SL, Schor AM and Rushton G: Fibroblasts from cancer patients display a mixture of both foetal and adult-like phenotypic characteristics. J Cell Sci 90: 401-407, 1988.

24. Reinertsen T, Halgunset J, Viset T, Flatberg A, Haugsmoen LL and Skogseth H: Gene expressional changes in prostate fibroblasts from cancerous tissue. APMIS 120: 558-571, 2012.

25. Cifone MG, Botti D, Festuccia C, Napolitano T, del Grosso E, Cavallo G, Chessa MA and Santoni A: Involvement of phospholipase A2 activation and arachidonic acid metabolism in the cytotoxic functions of rat NK cells. Cell Immunol 148: 247-258, 1993.

26. Ayala G, Tuxhorn JA, Wheeler TM, et al: Reactive stroma as a predictor of biochemical- free recurrence in prostate cancer. Clin Cancer Res 9: 4792-4801, 2003.

27. McAlhany SJ, Ayala GE, Frolov A, et al: Decreased stromal expression and increased epithelial expression of WFDC1/ps20 in prostate cancer is associated with reduced recurrence-free survival. Prostate 61: 182-191, 2004.

28. Orimo A, Gupta PB, Sgroi DC, et al: Stromal fibroblasts present in invasive human breast carcinomas promote tumor growth and angiogenesis through elevated SDF-1/CXCL12 secretion. Cell 121: 335-348, 2005.

29. Rowley DR: What might a stromal response mean to prostate cancer progression? Cancer Metastasis Rev 17: 411-419, 1998.

30. Tuxhorn JA, McAlhany SJ, Dang TD, Ayala GE and Rowley DR: Stromal cells promote angiogenesis and growth of human prostate tumors in a differential reactive stroma (DRS) xenograft model. Cancer Res 62: 3298-3307, 2002.

31. Thalmann GN, Rhee H, Sikes RA, Pathak S, Multani A, Zhau HE, Marshall FF and Chung LW: Human prostate fibroblasts induce growth and confer castration resistance and metastatic potential in LNCaP cells. Eur Urol 58: 162-171, 2009.

32. Goetz JG, Minguet S, Navarro-Lérida I, Lazcano JJ, Samaniego R, Calvo E, et al: Biomechanical remodeling of the microenvironment by stromal caveolin-1 favors tumor invasion and metastasis. Cell 146: 148-163, 2011.

33. Giatromanolaki A, Koukourakis MI, Koutsopoulos A, Mendrinos S and Sivridis E: The metabolic interactions between tumor cells and tumor-associated stroma (TAS) in prostatic cancer. Cancer Biol Ther 13: Nov 1, 2012 (Epub ahead of print).

34. Freeman MR, Yang W and Di Vizio D: Caveolin-1 and prostate cancer progression. Adv Exp Med Biol 729: 95-110, 2012.

35. Giannoni E, Taddei ML, Parri M, Bianchini F, Santosuosso M, Grifantini R, Fibbi G, Mazzanti B, Calorini L and Chiarugi P: EphA2-mediated mesenchymal-amoeboid transition induced by endothelial progenitor cells enhances metastatic spread due to cancer-associated fibroblasts. J Mol Med (Berl): Aug 19, 2012 (Epub ahead of print).

36. Franco OE, Shaw AK, Strand DW and Hayward SW: Cancer associated fibroblasts in cancer pathogenesis. Semin Cell Dev Biol 21: 33-39, 2010

37. Daja MM, Niu X, Zhao Z, Brown JM and Russell PJ: Characterization of expression of matrix metalloproteinases and tissue inhibitors of metalloproteinases in prostate cancer cell lines. Prostate Cancer Prostatic Dis 6: 15-26, 2003.

38. Wilson MJ, Sellers RG, Wiehr C, Melamud O, Pei D and Peehl DM: Expression of matrix metalloproteinase-2 and -9 and their inhibitors, tissue inhibitor of metalloproteinase- 1 and -2 , in primary cultures of human prostatic stromal and epithelial cells. J Cell Physiol 191: 208-216, 2002.

39. Zhang J, Jung K, Lein M, Kristiansen G, Rudolph B, Hauptmann S, Schnorr D, Loening SA and Lichtinghagen R: Differential expression of matrix metalloproteinases and their tissue inhibitors in human primary cultured prostatic cells and malignant prostate cell lines. Prostate 50: 38-45, 2002. 